typeset using JPSJ.sty $<$ ver.1.0b $>$

\title{
Magnetic Phase Diagram and Metal-Insulator Transition of $\mathrm{NiS}_{2-x} \mathrm{Se}_{x}$
}

\author{
Masato Matsuura*, Haruhiro Hiraka, Kazuyoshi Yamada** and Yasuo Endoh \\ Department of Physics, Tohoku University, Sendai 980-8578
}

(Received December 22,1999)

\begin{abstract}
Magnetic phase diagram of $\mathrm{NiS}_{2-x} \mathrm{Se}_{x}$ has been reexamined by systematic studies of electrical resistivity, uniform magnetic susceptibility and neutron diffraction using single crystals grown by a chemical transport method. The electrical resistivity and the uniform magnetic susceptibility exhibit the same feature of temperature dependence over a wide Se concentration. A distinct first order metal-insulator (M-I) transition accompanied by a volume change was observed only in the antiferromagnetic ordered phase for $0.50 \leq x \leq 0.59$. In this region, the M-I transition makes substantial effects to the thermal evolution of staggered moments. In the paramagnetic phase, the M-I transition becomes broad; both the electrical resistivity and the uniform magnetic susceptibility exhibit a broad maximum around the temperatures on the M-I transition-line extrapolated to the paramagnetic phase.
\end{abstract}

KEYWORDS: metal-insulator transition, mott-insulator, antiferromagnetism, neutron diffraction, phase diagram

\section{$\S 1$. Introduction}

$\mathrm{NiS}_{2}$ has been recognized for a long time as a prototype of Mott insulator described by the Mott-Hubbard narrow band picture. Hence, many experimental results on magnetism and transport properties in this system have been interpreted by this scenario, ${ }^{1)}$ where a metallic phase appears by a broadening of 3d-bands with either applying pressure beyond $\sim 3.5 \mathrm{GPa}$ or $\sim 25 \%$ substitution of $\mathrm{S}$ sites with Se atoms.

However, a recent photo-emission spectroscopy experiment revealed that subsequent change in the band structure upon Se-substitution should not be described by the band broadening but is governed by the charge-transfer within a band gap. ${ }^{2}$ ) Moreover, recent observations by the angle-resolved photoemission spectroscopy (ARPES) in the metallic phase near the phase boundary of metalinsulator transition (M-I boundary) showed a sharp peak in the spectrum near the Fermi energy. ${ }^{3,4)}$ Enhancement of the effective mass near the M-I boundary ${ }^{5)}$ also suggests a breakdown of the previous band picture.

An antiferromagnetic (AF) long-range order has been confirmed to exist in both metallic and insulating phases. ${ }^{6)}$ The AF long-range ordered structure in $\mathrm{NiS}_{2}$ is complicated showing two types of structure $\left(\mathbf{q}_{\mathrm{M} 1}=\right.$ (001) and $\mathbf{q}_{\mathrm{M} 2}=\left(\frac{1}{2} \frac{1}{2} \frac{1}{2}\right)$ on fcc lattice). ${ }^{7-10)}$ Although several studies on magnetic phase diagram of $\mathrm{NiS}_{2-x} \mathrm{Se}_{x}$ were carried out,, ,11-14) there still exist non-trivial discrepancies in the Néel temperature $\left(T_{\mathrm{N}}\right)$ and M-I transition temperature $\left(T_{\mathrm{MI}}\right)$. Furthermore, no systematic neutron study on $\mathrm{NiS}_{2-x} \mathrm{Se}_{x}$ with single crystal has been carried out.

We succeeded in growing a series of single crystals over a wide Se-concentration. Then, we performed a system-

\footnotetext{
* E-mail: matsuura@iiyo.phys.tohoku.ac.jp

** Present address: Institute for Chemical Research, Kyoto University, Gokasho, Uji, Kyoto 611-0011.
}

atic study to reexamine the magnetic phase diagram and the M-I transition itself, and established a new phase diagram of $\mathrm{NiS}_{2-x} \mathrm{Se}_{x}$ system with which an intimate relation between magnetic and transport properties was made it clear.

\section{§2. Experimental Detail}

Prescribed amount of $\mathrm{Ni}, \mathrm{S}$ and Se powders in $5 \mathrm{~N}$ purity were mixed with $3 \%$ of excess $\mathrm{S}$ and Se. They were sintered in an evacuated silica tube at $720^{\circ} \mathrm{C}$ for 7 days. In order to improve the homogeneity of starting powders, we repeated the above process of sintering and grinding twice. The Se-concentration of polycrystalline samples was determined from the lattice constant by utilizing Ve-

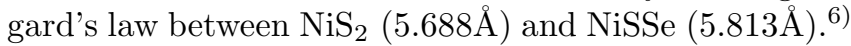

Then, single crystals were grown by a chemical transport method using $\mathrm{Cl}_{2}$ gas. ${ }^{15)}$ Polycrystalline powder of $0.5 \mathrm{~g}$ was sealed under 0.5 atm of $\mathrm{Cl}_{2}$ gas in an evacuated silica ampoule (10 $\phi$ in inner diameter and 14-20 $\mathrm{cm}$ in length) and was placed in a furnace. The average temperature was kept at about $750^{\circ} \mathrm{C}$ with the temperature gradient, $2^{\circ} \mathrm{C} / \mathrm{cm}$. Single crystals were grown up to $4 \mathrm{~mm}$ on an edge for a month of growth. They are characterized by shiny (100) and (111) facets.

The electrical resistivity $(\rho)$ was measured by the standard four-probe method between $4.2 \mathrm{~K}$ and room temperature. The uniform magnetic susceptibility $(\chi)$ was measured with a standard SQUID magnetometer under a magnetic field of $1 \mathrm{~T}$ from $5 \mathrm{~K}$ to $300 \mathrm{~K}$. Neutron diffraction measurements were performed on the tripleaxis spectrometer TOPAN installed at the JRR-3M Reactor of Japan Atomic Energy Research Institute. Incident and final neutron energy was fixed to be $14.7 \mathrm{meV}$ $\left(\lambda=2.67 \AA^{-1}\right)$ using pyrolytic graphite (PG) monochromator and analyzer. Horizontal collimation of the neutron beam was set to be 60'-30'-60'-100', from the forefront of the monochromater to the entrance of neutron detector. The sample was mounted so as to access to 
the $(h, h, l)$ reciprocal lattice plane. In this paper, we denote the indices of reflection in the AF unit cell which is twice as large as the chemical unit cell, as used in previous works. ${ }^{11)}$ Temperature dependence of lattice constant was measured by using a standard four-circle spectrometer with x-rays provided from a rotating anode $\left(40 \mathrm{kV} \times 100 \mathrm{meV}, \mathrm{MoK} \alpha_{1}\right)$ and monochromized using $\mathrm{PG}(002)$ reflection.

In order to investigate the M-I transition in detail, we performed simultaneous measurements on $\rho$ and staggered magnetization for $x=0.50$ and 0.53 by contacting copper wires for resistivity measurement onto the crystal mounted in an aluminum can for the neutron diffraction measurement. Intensities of $\mathrm{AF}(002)$ Bragg reflection (type I) and $\rho$ were monitored every 30 and 50 seconds, respectively. We changed the temperatures at a constant rate of $0.5 \mathrm{~K} / \mathrm{min}$.

\section{$\S 3 . \quad$ Experimental Results}

\subsection{Neutron diffraction}

Bragg reflections corresponding to type I AF structure were observed in both the insulator and the metallic phase $(x \geq 0.5)$, while reflections of type II AF structure vanish for $x>0.3$, which is consistent with the previous result. ${ }^{16)}$ Thermal evolutions of peak intensity of (002) AF Bragg reflection $I(T)$, as typical examples of type I, are shown in Figs. 1 (a) for $x=0.50$ and (b) for $x=0.69$.
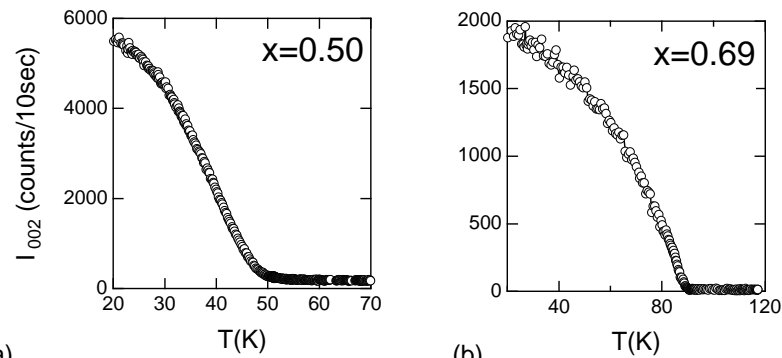

(a)

(b)

Fig. 1. Thermal variation of peak intensity of (002) antiferromagnetic Bragg reflection for $\mathrm{NiS}_{2-x} \mathrm{Se}_{x}$ (a) $x=0.50$ and (b) $x=0.69$

We determined both $T_{\mathrm{N}}$ and a critical exponent $\beta$ on the basis of eqs.(1) and (2), assuming a Gaussian distribution of $T_{\mathrm{N}}$.

$$
\begin{gathered}
I(T) \propto \begin{cases}\int\left(\frac{\left|T-T_{\mathrm{N}}\right|}{T_{\mathrm{N}}}\right)^{2 \beta} f\left(T_{\mathrm{N}}\right) \boldsymbol{d} T_{\mathrm{N}} & \left(T<T_{\mathrm{N}}\right) \\
0 & \left(T>T_{\mathrm{N}}\right),\end{cases} \\
f\left(T_{\mathrm{N}}\right)=\frac{1}{\sqrt{2 \pi} \sigma} \exp \left(-\frac{\left(T_{\mathrm{N} 0}-T_{\mathrm{N}}\right)^{2}}{2 \sigma^{2}}\right) .
\end{gathered}
$$

The least-square fitting gives $T_{\mathrm{N} 0}=45.0 \mathrm{~K}, \sigma=7.4$ $\mathrm{K}$ and $\beta=0.71$ for $x=0.50$, and $T_{\mathrm{N} 0}=89.4 \mathrm{~K}$, $\sigma=0.9 \mathrm{~K}$ and $\beta=0.39$ for $x=0.69$. The distribution of $T_{\mathrm{N}} \sigma\left(T_{\mathrm{N}}\right)$ can be converted into the distribution of Se-concentration $\sigma(x)$ in the sample by using the $x$ dependence of $T_{\mathrm{N}}$. The $\sigma(x)$ is determined to be $\sim 0.01$ for both $x=0.50$ and $x=0.69$. For samples with $x<0.44$ and $x>0.60$, the critical exponent $\beta$ is determined to be
$0.35 \sim 0.40$, which is consistent with the theoretical value of the three dimensional Heisenberg model (0.367). On the other hand, for samples with $0.44 \leq x \leq 0.59, \beta$ exceeds 0.5. We note that the unusually large $\beta$ is observed only for samples with $T_{\mathrm{N}}$ close to the M-I boundary.

Assuming the noncollinear type I AF structure, ${ }^{9,10}$ ) we calculated the magnitude of sublattice magnetic moment from the intensities of two AF (002), (220) and nuclear (222) reflections measured at $10 \mathrm{~K}$. We used the diffraction data from polycrystalline powder samples to minimize the uncertainty of extinction effect. The magnetic moment thus obtained is shown in Fig. 2. Our result almost reproduces previous one by Miyadai et al. ${ }^{16)}$ However, our data indicate a change in Se-concentration dependence around the M-I boundary; the staggered moment decreases more rapidly with Se-substitution in the metallic phase than in the insulating one.

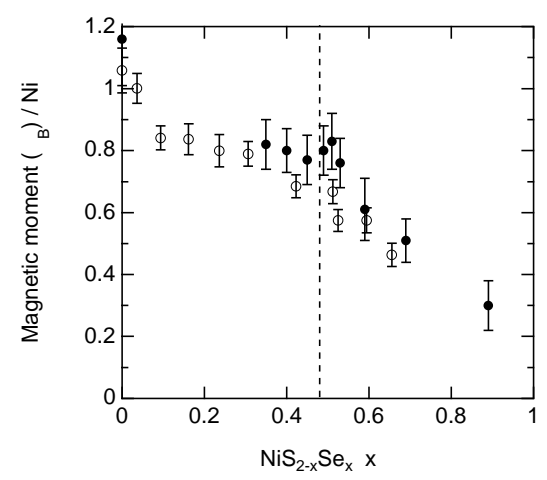

Fig. 2. $x$-dependence of the magnetic moment for $\mathrm{NiS}_{2-x} \mathrm{Se}_{x}$ at $10 \mathrm{~K}$. Closed and open circles represent present data and those by Miyadai et al. ${ }^{16)}$ respectively. Dotted line shows the M-I boundary at $0 \mathrm{~K}$.

\subsection{Electrical resistivity and uniform magnetic suscep- tibility}

The temperature dependences of $\rho$ and $\chi$ are shown in Figs. 3 and 4 , respectively. We note that both measurements were performed for the identical crystals. From thermal evolutions of $\rho$ and $\chi$, we can categorize four compositional regions in terms of M-I transition; (1) semiconducting region $(0 \leq x \leq 0.47)$, (2) first-order M-I transition $(0.50 \leq x \leq 0.59)$, (3) broad M-I transition $(x \sim 0.65)$, and (4) metallic region $(x \geq 0.69)$.

(1) Semiconducting region $(0 \leq x \leq 0.47)$

$\rho$ exhibits a typical feature of activation type for usual semiconductors. The activation energy $E_{\mathrm{a}}$ at room temperature decreases with increasing Se-substitution from $80 \mathrm{meV}\left(\mathrm{NiS}_{2}\right)$ to $50 \mathrm{meV}(x=0.47)$. These values are consistent with the hopping energies determined by Kwizera et $a l^{17}{ }^{17}$

$\chi$ shows a well-defined cusp corresponding to $T_{\mathrm{N}}$ determined by neutron diffraction measurements. $T_{\mathrm{N}}$ is almost constant at $40 \mathrm{~K}$ in the semiconducting region. For $\mathrm{NiS}_{2}$, a weak ferromagnetism appears below $T_{\mathrm{c}}=30$ $\mathrm{K}$. For samples with $0.36 \leq x \leq 0.47, T_{\mathrm{c}}$ drops down 


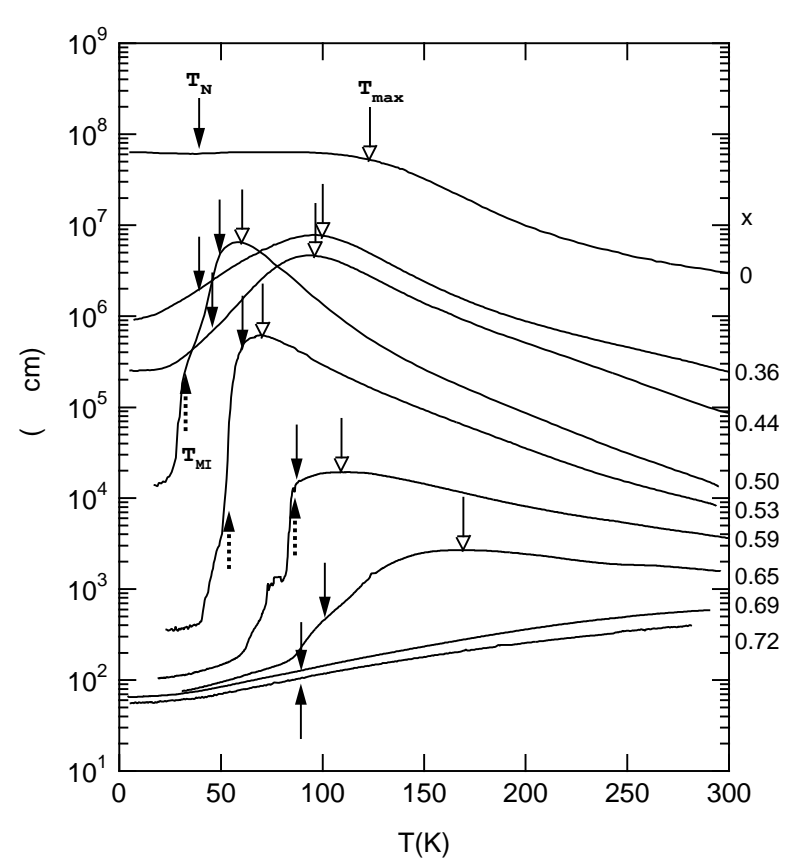

Fig. 3. Temperature dependences of the electrical resistivity $(\rho)$ for $\mathrm{NiS}_{2-x} \mathrm{Se}_{x}$ single crystals. Solid arrows indicate $T_{\mathrm{N}}$ determined by neutron diffraction measurements. Dashed arrows point at the temperature where $\rho$ drops discontinuously. Open arrows represent the temperature at which $\rho$ reaches the maximum.

to $20 \mathrm{~K}$ with the weak bulk ferromagnetization, which is $10^{-3}$ times smaller than that of $\mathrm{NiS}_{2}$.

$\rho$ exhibits a plateau or a broad maximum at $T_{\max }$ as indicated by open arrows in Fig. 3. $T_{\max }$ is higher than $T_{\mathrm{N}}$ and decreases with increasing $x$ from $120 \mathrm{~K}\left(\mathrm{NiS}_{2}\right)$ to $80 \mathrm{~K}(x=0.47) . \chi$ does not follow a simple Curie-Weiss law above $T_{\mathrm{N}}$. These facts suggest that the short-range magnetic correlation develops well above $T_{\mathrm{N}}$, strongly coupled with transport properties.

(2) First order M-I transition $(0.50 \leq x \leq 0.59)$

At high temperatures, $\rho$ shows an activation type behavior. $E_{a}$ determined at room temperature decreases with Se-substitution from $30 \mathrm{meV}(x=0.50)$ to $20 \mathrm{meV}$ $(x=0.59)$.

The whole process of the M-I transition in $\mathrm{NiS}_{2-x} \mathrm{Se}_{x}$ seems to occur in a broad temperatures range with a width of $20 \sim 30 \mathrm{~K}$. Both $\rho$ and $\chi$ exhibit a maximum (open arrows in Figs. 3 and 4 ) at temperatures higher than $T_{\mathrm{N}}$ (solid arrow), but no cusp appears at $T_{\mathrm{N}}$ in $\chi$. Below $T_{\mathrm{N}}, \rho$ rapidly decreases with decreasing temperature, and then further drops discontinuously by order of $10^{-1} \sim 10^{-2}$, indicating the first order M-I transition occurs in the AF ordered state. $T_{\mathrm{MI}}$ is defined as the temperature where $\rho$ discontinuously drops. The difference between $T_{\mathrm{MI}}$ and $T_{\mathrm{N}}$ becomes small with increasing $x$.

$\rho$ further decreases below $T_{\mathrm{MI}}$, and eventually exhibits a weak temperature-dependent metallic behavior. We have investigated the detailed feature of the M-I transition by simultaneous measurements on the electrical resistivity and the staggered magnetization, as described

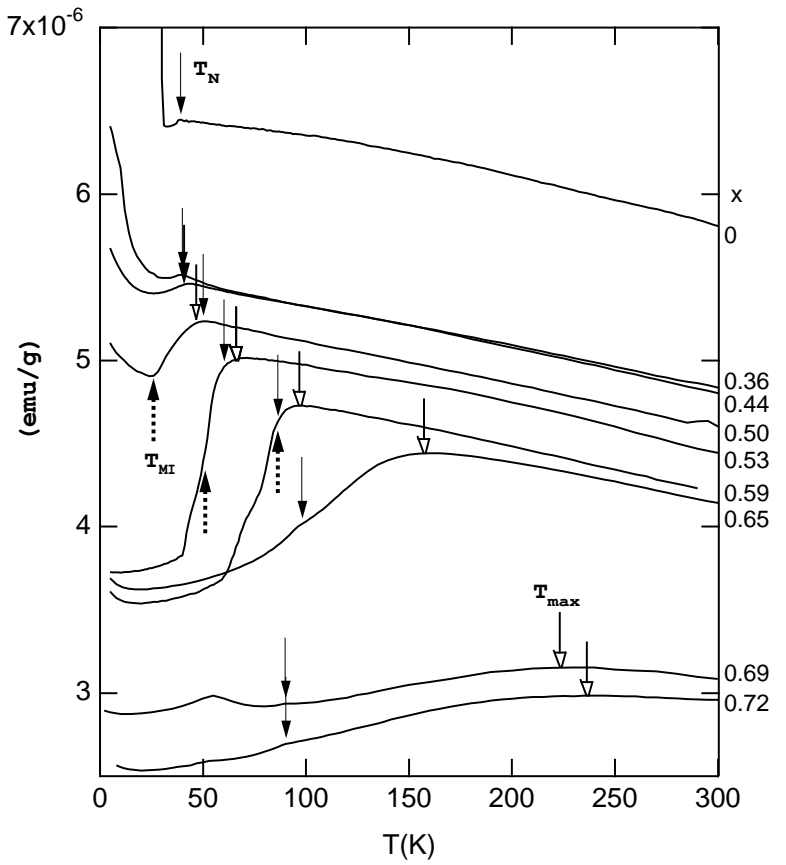

Fig. 4. Temperature dependences of the uniform magnetic susceptibility $(\chi)$ for $\mathrm{NiS}_{2-x} \mathrm{Se}_{x}$ single crystals. Solid and dashed arrows indicate $T_{\mathrm{N}}$ and $T_{\mathrm{MI}}$, as in Fig.3. Open arrows represent the temperature at which $\chi$ reaches the maximum.

in $\S 3.3$.

(3) Broad M-I transition $(x \sim 0.65)$

Although the phase transition from semiconductor to metal occurs on cooling, no discontinuous change was observed in $\rho$ and $\chi$. As shown for $x=0.65$ in Figs. 3 and 4 , both $\rho$ and $\chi$ exhibit a broad maximum in the paramagnetic phase; at the temperatures higher than $T_{\mathrm{N}}$ by about $60 \mathrm{~K}$. $T_{\max }$ increases with increasing $x$, while $T_{\mathrm{N}}$ saturates in this region. In contrast to the region (2), $\chi$ shows a small cusp at $T_{\mathrm{N}} . \rho$ exhibits metallic behavior at low temperatures. It is noted that this broad M-I transition crossovers to the following metallic one at the Se-concentration with the maximum $T_{\mathrm{N}}$.

(4) Metallic region $(x \geq 0.69)$

$\rho$ shows a typical metallic behavior below $300 \mathrm{~K}$ as shown in Fig. 3 for $x \geq 0.69$. However, a broad maximum in $\chi$ still exists well above $T_{\mathrm{N}}$ as indicated by open arrows in Fig. 4, which suggests the persistence of spin correlation at much higher temperatures above $T_{\mathrm{N}}$. Similar to the previous work, ${ }^{18)} T^{2}$ dependence in $\rho$ was observed at low temperatures. Crossover from $T^{2}$ to T-linear behavior occurs around temperatures where $\chi$ shows a maximum.

\subsection{Detailed simultaneous scan on electrical resistivity and neutron diffraction near M-I transition}

As described above, the first order M-I transition was observed for $0.50 \leq x \leq 0.59$ in the AF ordered phase. In order to study correlation between the M-I transition and the magnetism in more detail, we performed simultaneous measurements on the electrical resistivity and staggered magnetization; the peak height of (002) AF Bragg reflection $I_{002}$. Temperature dependences of $\rho$ and $I_{002}$ 
measured simultaneously are shown in Figs. 5 for (a) $x=0.50$ and (b) $x=0.53$ single crystals.

For both $x=0.50$ and $0.53, \rho$ sharply drops below $T_{\mathrm{N}}$ and the first order M-I transition occurs in the AF ordering state. For $x=0.59$ as shown in Fig. 5(c), both magnetic order and metallic phase appear almost simultaneously. As is clearly seen in Figs. 5, there exists an unusual correlation between the staggered magnetization and electrical resistivity. $\rho$ gradually decreases associated with tailing in $I_{002}$ around $T_{\mathrm{N}}$, which gives unusually large critical exponent $\beta$. In addition, $I_{002}$ show a small but clear kink at $T_{\mathrm{MI}}$ where $\rho$ drastically change in Figs. 5(b) and 5(c). Moreover, there exists an unusual kink in $I_{002}$ at temperature below which $\rho$ and $\chi$ show metallic behavior. Below the kink temperature, $T_{\text {kink}}$, temperature dependence of $I_{002}$ becomes weak in metallic phase. This phenomenon is not due to the extinction for the single crystal, since the same phenomenon was also observed for $x=0.53$ in the polycrystalline powder sample.

(a)

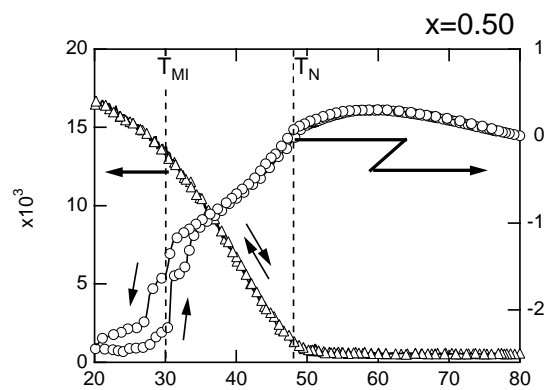

(b)

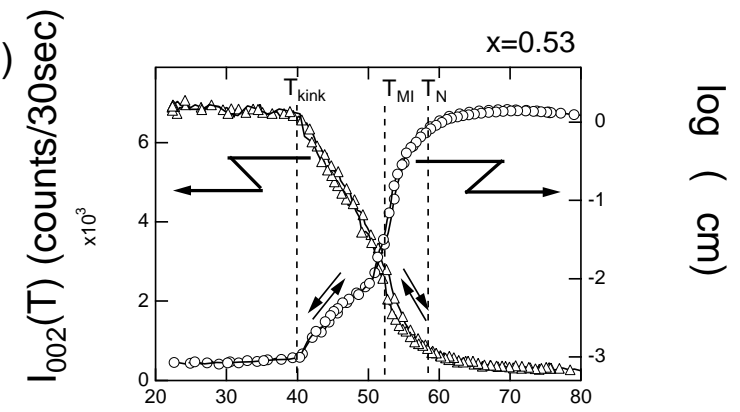

(c)

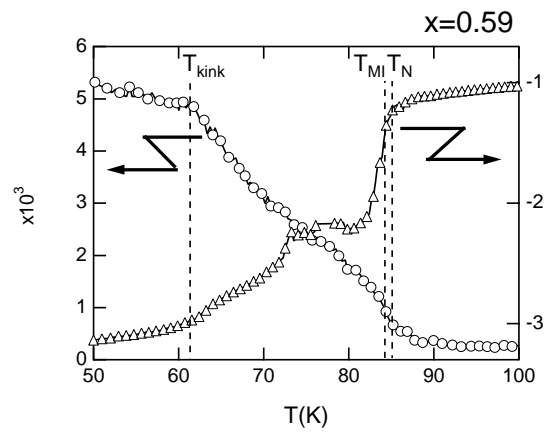

Fig. 5. Temperature dependences of the electrical resistivity and the peak intensity of (002) antiferromagnetic Bragg reflection, $I_{002}(T)$ measured simultaneously for (a) $x=0.50$ and (b) $x=0.53$, and separately for (c) $x=0.59$. Circles and triangles indicate electrical resistivity and $I_{002}(T)$, respectively.

It is noted $\rho$ shows a multi step change near the M-I transition as shown in Figs. 5. Here we describe on the multi-step like change in $\rho$. For the data from $x=0.50$, the multi step change near $30 \mathrm{~K}$ in Fig. 5(a) was concluded to be ascribed in the following extrinsic reason. The single crystals of $x=0.50$ were grown from a mixing of two powder samples synthesized separately, which gives rise to a slightly difference in concentration. After this simultaneous measurement, we synthesized the polycrystalline powder sample of $x=0.50$ and grew again the single crystal which shows a single step change at the MI transition. Therefore, we concluded the single crystal of $x=0.50$ showing two step anomalies contained two regions with slightly different Se-concentration by about $x=0.005$

However, for $\mathrm{x}=0.53$ and 0.59 we found no distinct experimental reason to induce inhomogeneity of Seconcentration. The same multi step change of $\rho$ was also observed in a previous independent study on single crystals grown by a flux method. ${ }^{19)}$ Therefore, the multistep change for $\mathrm{x}=0.53$ and 0.59 can be intrinsic unlike the present data for $x=0.50$. We only speculate that between $T_{\text {kink }}$ and $T_{\mathrm{MI}}$, the metallic and the insulating phases possibly coexist due to the first-order transition at $T_{\mathrm{MI}}$. The multi step change of $\rho$ may relate with such an inhomogeneous state in this temperature region.

As shown in Fig. 6, the lattice constant discontinuously changes with a small thermal hysteresis only at the M-I transition, but no anomaly at $T_{\text {kink }}$. The small difference in magnitude of lattice constant between Fig.6 and its inset is due to the slight shift in position of sample, $0.02^{\circ}$, on cooling process. This first order M-I transition can be interpreted to be associated with the volume contractions with decreasing temperature. Applying the Clausius-Clapeyron relation to the present data, entropy change at the M-I transition $\Delta S$ is calculated to be $1.6( \pm 0.3)[\mathrm{J} /(\mathrm{mol} \mathrm{deg})$.$] , as previous report of M-I$ transition induced by pressure. ${ }^{20)}$ This value is in good agreement with that from the specific heat measurement by Sudo et al., $\Delta S=1.0[\mathrm{~J} /(\mathrm{mol} \mathrm{deg.})]$ for $x=0.51$ polycrystalline powder sample. ${ }^{21)}$

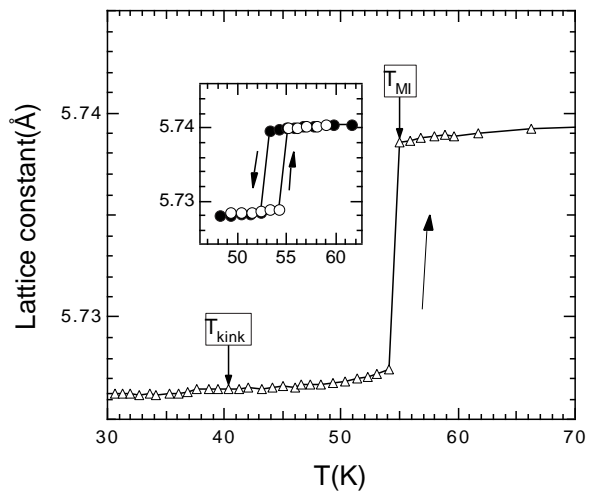

Fig. 6. Temperature dependence of lattice constant for $x=0.53$. Open and closed circles represent data on heating and cooling processes, respectively. 


\section{$\S 4 . \quad$ Discussion}

In Fig. 7, we present a revised phase diagram of $\mathrm{NiS}_{2-x} \mathrm{Se}_{x}$ as the summary of the present study. Here, we are mainly concerned about the phases near the MI phase boundary for $x>0.3$. Compared with previous results of the phase diagram, ${ }^{6,11-14)}$ we add two crossover regions as shown in Fig. 7. In the lower Seconcentration range, the crossover region depicted by vertical lines in the figure is determined by the temperature variation of transport properties. For example, $\rho$ exhibits a broad maximum at the crossover region. Such a crossover was also observed in thermoelectric power measurements. ${ }^{17,19)}$ Furthermore, our recent neutron inelastic scattering data for $\mathrm{NiS}_{2}$ show that the short-range magnetic correlation remains above $T_{\mathrm{N}}$ at least up to $\sim$ $200 \mathrm{~K}$. Therefore, we conjecture that the crossover behavior in the transport properties is inherently correlated with the magnetic short-range correlation. We call the region below the crossover as 'short-range antiferromagnetic insulating (AFI)' phase. The onset of short-range AFI phase decreases with increasing $x$, while $T_{\mathrm{N}}$ is almost constant in the insulating phase.

Another crossover in the higher Se-concentration range approximately corresponds to the M-I transition-line extrapolated towards the paramagnetic phase. Around the crossover temperature $\rho$ and $\chi$ show a broad peak. For $x \geq 0.69$, the peak in $\rho$ seems to disappear. For $0.50 \leq x \leq 0.59$, the peak appears at temperature slightly higher than that of the first order M-I transition. Here, we add a new phase called anomalous metal between $T_{\mathrm{N}}$ and $T_{\max }$. In Fig. 7 , we refer the data by Ogawa for $x \geq 1.0 .^{22}$ ) We note that such a broad maximum in $\chi$ was also observed in the paramagnetic phase of $\mathrm{La}_{2-x} \mathrm{Sr}_{x} \mathrm{CuO}_{4}$, high temperature superconducting cuprates. ${ }^{23)}$

A detailed magnetic phase diagram near the M-I boundary is shown in Fig. 8. We confirmed that the first order M-I transition occurs only in the AF ordered phase for $0.50 \leq x \leq 0.59$ from the simultaneous scan on $\rho$ and neutron diffraction, and the M-I transition becomes second order in the paramagnetic phase, as is seen in $\rho$ and $\chi$. According to the recent band calculation by the two-band Hubbard model in infinite dimension, a first order M-I transition in long range AF ordered phase is expected to occur in consideration of the electron hopping between nearest neighbor $\mathrm{Ni} 3 \mathrm{~d}$ and ligand $3 \mathrm{p} .{ }^{24}$ ) It suggests the charge-transfer nature being indispensable to understand the mechanism of M-I transition in this system. We note that the charge-transfer nature is seen in ARPES spectrum as a sharp peak near the Fermi energy in the metallic phase around the M-I boundary.

It is important to compare the M-I transition in $\mathrm{NiS}_{2-x} \mathrm{Se}_{x}$ studied here with the previous experiment under pressure for $\mathrm{NiS}_{2}$. Both phase diagrams under pressure and Se-substitution are qualitatively the same with each other. ${ }^{25)}$ However, there exists an important difference between the two. In fact, a recent electrical resistivity measurement revealed that the first order M-I transition in $\mathrm{NiS}_{2}$ occurs at $150 \mathrm{~K}$ at $3.0 \mathrm{GPa},{ }^{26)}$ which

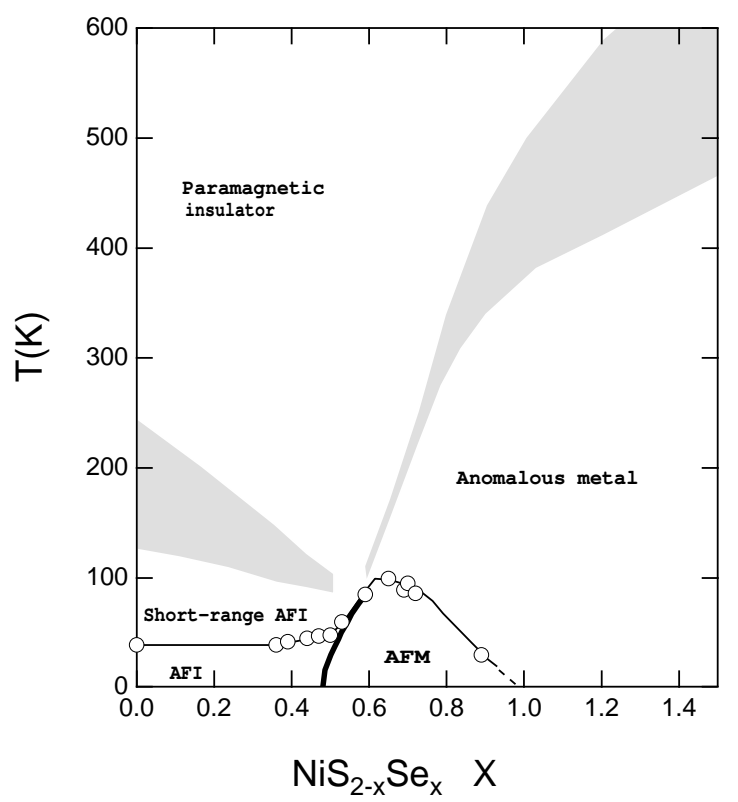

Fig. 7. Magnetic phase diagram of $\mathrm{NiS}_{2-x} \mathrm{Se}_{x}$. Open circles represent $T_{\mathrm{N}}$. Bold line shows a line of first order M-I transition (see Fig. 8 for more detail). Lines are guide to the eyes. The region depicted by vertical lines indicate a crossover region where transport properties or $\chi(T)$ reach a maximum; $\rho(T)$ and Seebeck coefficient $^{17,19)}$ for $x \leq 0.47, \rho(T)$ and $\chi(T)$ for $0.50 \leq x \leq 0.65$, and $\chi(T)$ for $x \geq 0.69$. For $x \geq 1.0$, we referred the data by Ogawa. ${ }^{22)}$ AFI and AFM denote antiferromagnetic insulator and metal, respectively.

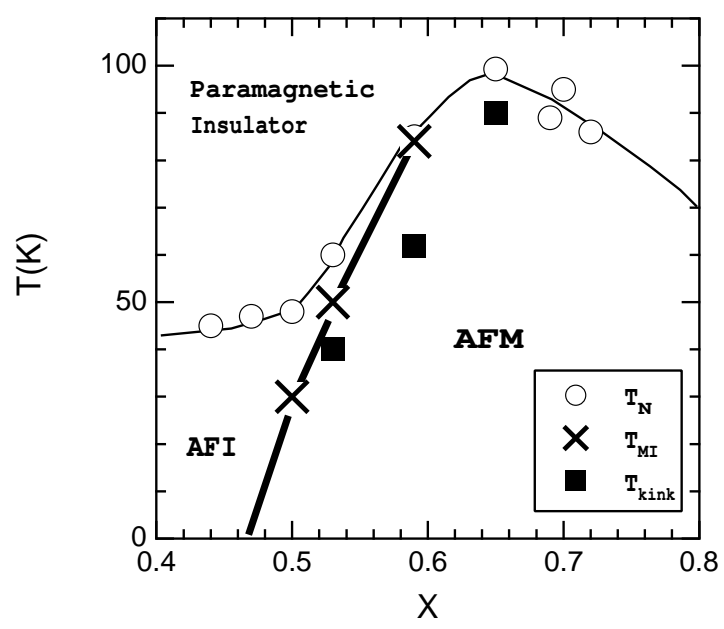

Fig. 8. Detailed magnetic phase diagram near M-I boundary. Solid squares point at temperatures where $\rho(T)$ shows a kink. Well-defined first order M-I transition was observed at $T_{\mathrm{MI}}$, represented by $\times$. 
can be converted into $x \sim 0.6$ for Se-substitution. On the other hand for Se-substitution, the maximum temperature for both the first order M-I transition and the long range AF ordering are limited to $100 \mathrm{~K}$. Therefore, the observation of the M-I transition at $150 \mathrm{~K}$ under pressure may conflict with our conjecture that the first order M-I transition occurs only in the antiferromagnetic phase. We speculate however, local randomness by Se-substitution may reduce the transition temperatures. Therefore, it is highly required to determine the $T_{\mathrm{N}}$ under high pressure beyond $\sim 3.5 \mathrm{GPa}$ for $\mathrm{NiS}_{2}$.

The revised phase diagram in $\mathrm{NiS}_{2-x} \mathrm{Se}_{x}$ is quite similar to those of other strongly correlated electron systems, such as $\mathrm{V}_{2} \mathrm{O}_{3},{ }^{27}$ ) and organic conductors (BEDT$\mathrm{TTF})_{2} \mathrm{X},{ }^{28-30)}$ where antiferromagnetism and M-I transition are closely connected. In the latter material, a superconducting phase appears, while $\mathrm{V}_{2} \mathrm{O}_{3}$ and $\mathrm{NiS}_{2-x} \mathrm{Se}_{x}$ become AF metals.

In these systems, it is often observed that the onset temperature of long range ordered state is significantly suppressed compared to the thermal robustness of short-range magnetic correlation. This is the case for $\mathrm{NiS}_{2-x} \mathrm{Se}_{x}$. Since $\mathrm{Ni}^{2+}$ ions form an fcc lattice in this structure, the nearest neighbor spins can be magnetically frustrated. In fact, preliminary inelastic neutron scattering measurement on this system found an unusual magnetic excitations possibly due to this frustration effect.

\section{$\S 5$. Summary}

$\mathrm{NiS}_{2-x} \mathrm{Se}_{x}$ system has been reinvestigated systematically by neutron diffraction, electrical resistivity, uniform magnetic susceptibility and X-ray diffraction using both single crystals and powder samples. We revealed a clear correlation between the transport and magnetic properties in this system. Both the magnitude of magnetic moment and its temperature dependence are affected by electric transport properties, particularly by the M-I transition. A well-defined first order M-I transition was found to occur only in the antiferromagnetic state. On the other hand, the transition in the paramagnetic phase becomes broad and is seen in the resistivity up to the Se-concentration where $T_{\mathrm{N}}$ exhibits a maximum. The novel feature in the newly proposed magnetic phase diagram is the existence of two cross-over regions, one from short ranged AF phase to paramagnetic insulator (PI) and the other from anomalous metal to PI. These crossover features suggest that the short-range magnetic correlation remains well above $T_{\mathrm{N}}$ in both phases, which cannot be interpreted by a single Mott Hubbard model, but by a new model taking account of the strong electron correlations.

\section{Acknowledgements}

We would like to thank T. Takahashi and M. Onodera for their assistance in crystal growth, and H. Kimura, K. Hirota and K. Nemoto for their technical assistance in $\mathrm{x}$-ray diffraction measurements and neutron scattering. This work was supported by Research Fellowships of the Japan Society for the Promotion of Science for Young Scientists.
1) J. A. Wilson: The metallic and non-metallic states of matter (Taylor \& Francis, 1985) Chap.9.

2) A. Fujimori, K. Mamiya, T. Mizokawa, T. Miyadai, T. Sekiguchi, H. Takahashi, N. Môri and S. Suga: Phys. Rev. B 54 (1996) 16329.

3) A. Y. Matsuura, Z.-X. Schen, D. S. Dessau, C.-H. Park, T. Thio, J. W. Bennett and O. Jepsen: Phys. Rev. B 53 (1996) 7584 .

4) A. Y. Matsuura, H. Watanabe, C. Kim, S. Doniach, Z.-X. Shen, T. Thio and J. W. Bennett: Phys. Rev. B 58 (1998) 3690.

5) M. Imada, A. Fujimori and Y. Tokura: Rev. Mod. Phys. 70 (1998) 1039.

6) F. Gautier, G. Krill, M. F. Lapierre, P. Panissod, C. Robert, G. Czjzek, J. Fink and H. Schmidt: Phys. Lett. 53A (1975) 31.

7) J. M. Hasting and L. M. Corliss: IBM J. Res. Dev. 14 (1970) 227.

8) T. Miyadai, K. Takizawa, H. Nagata, H. Ito, S. Miyahara and K. Hirakawa: J. Phys. Soc. Jpn. 38 (1975) 115.

9) K. Kikuchi, T. Miyadai, T. Fukui, H. Ito and K. Takizawa: J. Phys. Soc. Jpn. 44 (1978) 410.

10) K. Kikuchi, T. Miyadai, H. Ito, and T. Fukui: J. Phys. Soc. Jpn. 45 (1978) 444.

11) S. Sudo: J. Magn. Magn. Mater. 114 (1992) 57.

12) X. Yao, Y. K. Kuo, D. K. Powell, J. W. Brill and J. M. Honig: Phys. Rev. B 56 (1997) 7129.

13) H. S. Jarrett, R. J. Bouchard, J. L. Gillson, G. A. Jones, S. M. Marcus and J. F. Weiher: Mater. Res. Bull. 8 (1973) 833.

14) G. Czjzek, J. Fink, H. Schmidt, G. Krill, M. F. Lapierre, P. Panissod, F. Gautier and C. Robert: J. Magn. Magn. Mater. 3 (1976) 58.

15) R. J. Bouchard: J. Cryst. Growth 2 (1968) 40.

16) T. Miyadai, S. Sudo, Y. Tazuke, N. Môri and Y. Miyako: J. Magn. Magn. Mater. 31-34 (1983) 337.

17) P. Kwizera, M. S. Dresselhaus and D. Adler: Phys. Rev. B 21 (1980) 2328.

18) S. Ogawa : Physica 86-88B (1977) 997.

19) X. Yao, J. M. Honig, T. Hogan, C. Kannewurf and J. Spalek: Phys. Rev. B 54 (1996) 17469.

20) N. Môri and T. Watanabe: Solid State Commun. 27 (1978) 567.

21) S. Sudo, T. Nishioka, Y. Miyako and T. Miyadai: J. Phys. Soc. Jpn. 55 (1986) 1806.

22) S. Ogawa: J. Appl. Phys. 223 (1979) 2308.

23) T. Nakano, M. Oda, C. Manabe, N. Momono, Y. Miura and M. Ido: Phys. Rev. B 49 (1994) 16000.

24) H. Watanabe and S. Doniach: Phys. Rev. B 57 (1998) 3829.

25) N. Môri and H. Takahashi: J. Magn. Magn. Mater. 31-34 (1983) 335.

26) Y. Sekine, H. Takahashi, N. Môri, T. Matsumoto and T. Kosaka: Phys. B 237-238 (1997) 148.

27) D. B. McWhan, T. M. Rice and J. P. Remeika: Phys. Rev. Lett. 23 (1969) 1384.

28) K. Murata, M. Ishibashi, Y. Honda, N. A. Fortune, M. Tokumoto, N. Kinoshita and H. Anzai: Solid State Commun. 76 (1990) 377.

29) J. E. Schirber, D. L. Overmyer, K. D. Carlson, J. M. Williams, A. M. Kini, H. H. Wang, H. A. Charlier, B. J. Love, D. M. Watkins and G. A. Yaconi: Phys. Rev. B 44 (1991) 4666.

30) Yu. V. Sushko, H. Ito, T. Ishiguro, S. Horiuchi and G. Saito: Solid State Commun. 87 (1993) 997. 